\title{
Proapoptotic Bid binds to monolysocardiolipin, a new molecular connection between mitochondrial membranes and cell death
}

\author{
M Degli Esposti ${ }^{*}$, IM Cristea ${ }^{2}$, SJ Gaskell ${ }^{1}$, Y Nakao ${ }^{1}$ and \\ C Dive ${ }^{1}$ \\ 1 School of Biological Sciences, University of Manchester, Oxford Road, \\ Manchester M13 9PT, UK \\ ${ }^{2}$ Michael Barber Centre for Mass Spectrometry, UMIST, Manchester, UK \\ * Corresponding author: M Degli Esposti, School of Biological Sciences, \\ University of Manchester, Stopford Building, Oxford Road, Manchester M13 \\ 9PT, UK. Tel: + 44-161-2757436; Fax: + 44-161-2755600; \\ E-mail: mauro.esposti@man.ac.uk
}

Received 13.3.03; revised 23.6.03; accepted 23.6.03; published online 1 August 2003 Edited by $\mathrm{G}$. Kroemer

\begin{abstract}
Recent evidence indicates that the mitochondrial lipid cardiolipin may be instrumental in the proapoptotic action of $\mathrm{Bcl}-2$ family proteins on mitochondrial membranes, leading to the release of apoptogenic factors. However, contrasting evidence indicates that progressive loss of cardiolipin occurs during apoptosis. Here we show that Bid, a crucial proapoptotic protein that integrates the action of other $\mathrm{Bcl}-2$ family members, exhibits discrete specificity for metabolites of cardiolipin, especially monolysocardiolipin (MCL). MCL, normally present in the remodelling of mitochondrial lipids, progressively increases in mitochondria during Fas-mediated apoptosis as a by-product of cardiolipin degradation, and also enhances Bid binding to membranes. MCL may thus play a crucial role in connecting lipid metabolism, relocation of Bid to mitochondria and integrated action of $\mathrm{Bcl}-2$ proteins on mitochondrial membranes. We propose that Bid interaction with MCL 'primes' the mitochondrial outer membrane via segregation of lipid domains, facilitating membrane discontinuity and leakage of apoptogenic factors.

Cell Death and Differentiation (2003) 10, 1300-1309, doi:10.1038/

sj.cdd. 4401306

Published online 1 August 2003
\end{abstract}

Keywords: apoptosis/Bid/mitochondria/cardiolipin

Abbreviations: MCL, monolysocardiolipin; $\mathrm{CL}$, cardiolipin; MS, mass spectroscopy; LPA, lysophosphatidic acid

\section{Introduction}

Proapoptotic proteins of the $\mathrm{Bcl}-2$ family regulate the pathways of apoptosis that induce the release of mitochondrial apoptogenic factors like cytochrome $c$, which once present in the cytoplasm activate the caspase cascade of cell degradation. ${ }^{1,2} \mathrm{Bid}$, a widespread proapoptotic member of the $\mathrm{Bcl}-2$ family, has attracted increasing interest since it was identified as a substrate of caspase 8 in pathways of apoptosis mediated by death receptors like Fas. ${ }^{3,4}$ Caspase cleavage of full-length (f.l.) Bid produces p15 tBid, which is usually considered the active form, ${ }^{2-4}$ even though it remains unclear as to how proteolysis increases the proapoptotic action of this protein. $^{5}$

At the mitochondrial surface, Bid integrates with other Bcl-2 family proteins, Bax or Bak, ${ }^{1,6}$ to produce physicochemical changes in mitochondrial membranes. ${ }^{1,7-14}$ Cardiolipin (CL), a lipid characteristic of mitochondria, has been reported to target tBid to mitochondria. ${ }^{8,13}$ This observation, despite contrasting results, ${ }^{15}$ has increased current awareness of the role of cardiolipin in the mechanism of membrane permeabilisation by proapoptotic Bcl-2 proteins. ${ }^{1,16,17}$ However, other evidence has shown that mitochondrial cardiolipin decreases during apoptosis induced by a variety of stimuli, ${ }^{18-20}$ thus creating a paradox. If Bid and Bax, which are predominantly cytosolic proteins, require cardiolipin for their action on mitochondria, ${ }^{1,13,17}$ how can they relocate to apoptotic mitochondria, which contain reduced levels of the same lipid? Using independent approaches, we provide evidence contributing a solution to this apparent paradox. For the first time, we demonstrate that Bid can bind to metabolites of cardiolipin like MCL and with increased affinity after caspase cleavage. We then show that MCL, unique among the lipids and surfactants tested, enhances the membrane association of both f.I. Bid and tBid, and at the same time stimulates the cytochrome $c$ releasing capacity of tBid in vitro. Finally, we report that MCL increases in vivo during Fas-induced apoptosis, a pathway of cell death that requires Bid for engaging mitochondria and their apoptogenic factors.

We discuss how the preferential interaction of tBid with $\mathrm{MCL}$, a membrane lipid normally present as part of the metabolic cycle of mitochondrial lipids, ${ }^{21,22}$ may constitute a key molecular connection between upstream stimuli of cell death and mitochondrial membranes.

\section{Results}

\section{Recombinant Bid contains bound lysolipids}

To provide a better understanding of the action of Bid, we have focused our study on its interaction with membrane lipids, a prerequisite for the alteration in mitochondrial membranes that is instrumental for the release of apoptogenic factors. ${ }^{1,2}$ 
Previous reports have implied an interaction of Bid and tBid with membrane lipids, ${ }^{5,7-9}$ but evidence for binding to specific lipids, besides to other $\mathrm{Bcl}-2$ proteins, has been limited and controversial (cf. Lutter et al.; ${ }^{8}$ Kuwana et al.; ${ }^{13}$ Newmeyer et al.; ${ }^{1}$ Zha et al. ${ }^{15}$ ). We thus decided to follow multiple approaches that could provide independent information documenting the binding of isolated Bid to selected lipids. We present these approaches with a progression from the simplest in vitro system to more complex systems including isolated mitochondria, and finally to an in vivo model of apoptosis in which Bid is crucially involved.

Following earlier studies on lipid transfer, ${ }^{9}$ we happened to note that recombinant Bid contained lipid material. In particular, mass spectroscopy (MS) analysis indicated that preparations of recombinant mouse Bid expressed in Escherichia coli predominantly contained a bound lysophosphatidic acid (LPA) with a long unsaturated acyl chain (18:1, Figure 1). This bound lipid obviously originated from the bacterial expressor, where LPA species containing 18: 1 acyl chains are present, albeit in low abundance - $E$. coli phospholipids mainly contain shorter and saturated acyl chains. ${ }^{20}$ Since bound LPA was present in preparations of Bid that were obtained by different procedures, we further excluded the fact that it could be derived from spurious contaminations.

Mammalian proteins with lipid transfer activity like $\mathrm{Bid}^{9}$ have been reported to retain E.coli-derived lipids after purification from bacteria. For instance, rat phosphatidylinositol transfer protein (PITP), once expressed in recombinant form, contains stoichiometric levels of phosphatidylglycerol $(P G)^{23}-$ a major $E$.coli lipid resembling phospholipids that are abundant in mammalian cells. ${ }^{20}$ We thus suspected that the presence of a minor bacterial lysolipid bound to recombinant Bid may reflect a 'natural selection' for some lipid intrinsically recognised by the protein within bacterial or mammalian cells. In particular, association to bacterial lipids suggested a potential specificity for cardiolipin metabolites, given the bacterial origin of eukaryotic mitochondria and the presence of long-chain LPA species in mammalian cells as precursors or by-products of CL. ${ }^{22}$ In line with this possibility, detailed MS analysis of mammalian cardiolipin showed the presence of LPA-related by-products (Figure 1c)

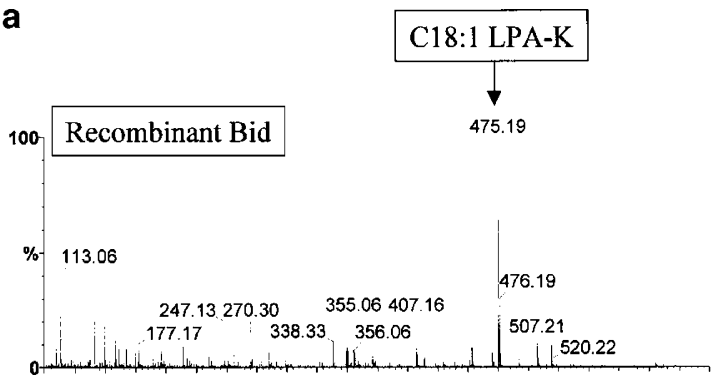

b

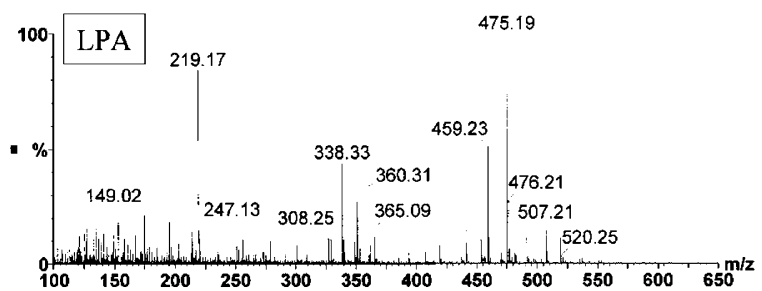

C
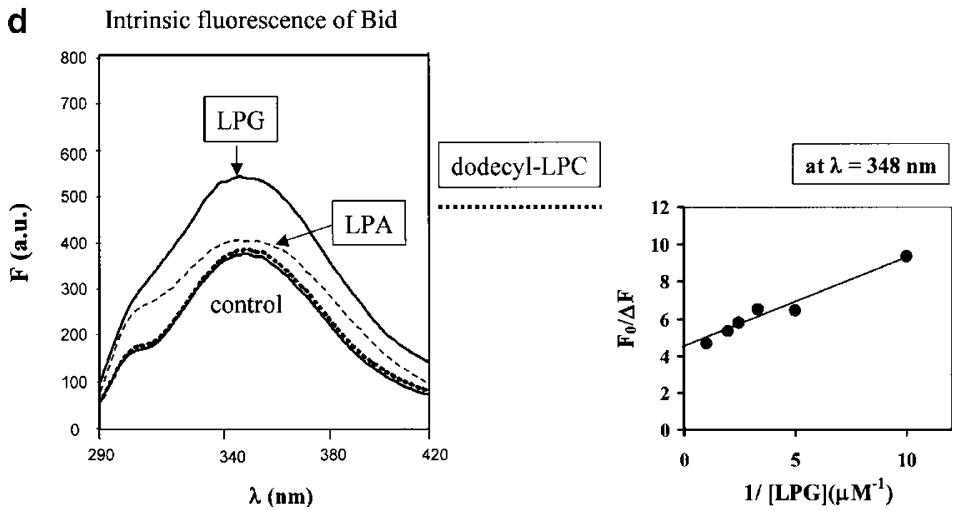

Figure 1 Recombinant Bid binds to lysolipids. (a) ES-MS analysis was performed in positive mode (cf. Petkovic et al. ${ }^{40}$ ) with recombinant f.I. Bid initially dissolved in KHepes $10 \mathrm{mM}, 100 \mathrm{mM} \mathrm{KCl}, \mathrm{pH} 7.4$ after purification from E.coli. The analytical spectrum was obtained with $4 \mathrm{pmol}$ of a fresh preparation after desalting with a zip-tip column; data are representative of $n=5$ different samples. (b) MS spectrum of standard 1-oleyl-LPA (predominant potassium salt) in the same medium as in (a). (c) MS spectrum of a typical commercial preparation of cardiolipin from beef heart, dissolved in ethanol. Note that the peaks with mass around 400 , which are present also in Bid samples (cf. (a)), consist of linoleoyl-containing fragments related to LPA (e.g. species with $\mathrm{m} / \mathrm{z}=413$ and 421 ). (d) Changes in the intrinsic fluorescence of recombinant Bid (dissolved at $0.8 \mu \mathrm{M}$ in assay buffer) were induced by binding to either LPA (dashed spectrum) or LPG (top solid spectrum) at $5 \mu \mathrm{M}$. Equivalent concentrations of nonionic detergents like OG or short-chain lysolipids like 1-dodecyl LPC (LPC-C12, dotted spectrum in the figure) did not produce significant changes in the intrinsic fluorescence of Bid, thereby excluding a nonspecific detergent effect. The bottom panel shows a representative plot of the changes in intrinsic fluorescence of Bid induced by increasing concentrations of LPG in solution. These data are plotted in double-reciprocal form, where $F_{0} / \Delta F$ is the ratio between the unquenched fluorescence and the fluorescence change measured at any ligand concentration, and the ratio between slope and intercept can be used to estimate the $K_{\mathrm{d}}$ for ligand quencher. ${ }^{42}$ Using such an estimation and linear regression, a $K_{d}$ of $0.087 \mu \mathrm{M}$ was obtained, which was close to the mean of values obtained with this method combined with that based on ADIFAB measurements $(0.086 \pm 0.003 \mu \mathrm{M}, n=4)$. 


\section{Bid binding to lipids in solution}

The unexpected finding of bacterial lipids bound to recombinant Bid (Figure 1a) prompted us to reproduce Bid binding to selected lipids in model systems. We first adopted the direct approach of measuring changes in the intrinsic fluorescence of the protein after the addition of pure lipids in solution. Similarly to established lipid-binding proteins, ${ }^{24,25}$ the intrinsic fluorescence of Bid changed in response to selected lysolipids, as shown in Figure 1d. Among the lysolipids tested, 1-oleyl-lysophosphatidylglycerol (LPG) induced the strongest increase in Bid fluorescence (Figure 1d), while other lysolipids like 1-dodecyl-lysophosphatidylcholine (dodecyl-LPC, dotted line in Figure 1d) and also nonionic detergents like octylglucoside (OG, not shown) had negligible effects. With LPG and other lysolipids, the fluorescence changes followed saturation behaviour allowing estimation of binding parameters, as shown in the bottom panel of Figure 1d. These results provided the first evidence for specificity in Bid binding to phospholipids, but were necessarily limited by the water solubility of the tested lipids. In fact, the turbidity produced by poorly soluble lipids such as $\mathrm{CL}$ and its immediate metabolites affected the measurements of intrinsic fluorescence and prevented an accurate evaluation of binding parameters (results not shown).

To minimise problems related to lipid solubility, we developed alternative approaches that provided accurate binding information. One approach exploited the properties of $A D I F A B$, a fatty acid-binding protein with a fluorescent reporter that responds to fatty acids ${ }^{26}$ and also to lysolipids, similarly to analogous probes. ${ }^{27}$ We found that the fluorescence of ADIFAB progressively decreased upon increasing the concentration of various lysolipids (and other surfactants) in their monomeric state and also in their minimal micellar state (i.e. the first stable aggregation that is reached upon exceeding the critical micellar concentration). Although $A D I F A B$ changes were less pronounced with the micellar than the monomeric state of lysolipids, interference by micelle turbidity was limited and could be independently evaluated using nonbinding di-acyl lipids (e.g. PG). Consequently, in many cases micellar lysolipids induced ADIFAB changes that could be analysed with the procedure described here allowing the evaluation of binding constants; these were often about one order of magnitude higher than those obtained with the same lipids in monomeric state. The approach could thus be applied also to CL metabolites like MCL that were predominantly nonmonomeric at the concentrations used (Figure 2a). Since these lipids are nonmonomeric also within natural membranes, ${ }^{22}$ the binding parameters obtained with the ADIFAB approach might be of relevance to the physiological situation.

We determined first the $K_{d}$ of ADIFAB for a variety of lysolipids by using either the ratiometric analysis described previously ${ }^{26}$ or a numerical solution derived thereof (see Materials and Methods). The $K_{\mathrm{d}}$ values obtained for oleate and various lysolipids were in fair agreement with those reported earlier, ${ }^{26,27}$ thereby validating our procedure. We then performed ADIFAB measurements with all lysolipids related to $\mathrm{CL}$ metabolism in the absence and presence of f.l. Bid or caspase 8-cleaved Bid (tBid, Figure 2a). When these proteins were added to the system, ADIFAB fluorescence became less sensitive to the addition of lysolipids (Figure 2a), suggesting a competition between Bid and ADIFAB for ligand binding. This competition was effectively decreasing the concentration of free lysolipid in solution, so that at any point in the titration ADIFAB quenching was less pronounced than in the absence of Bid (cf. Figure 2a, left). Conversely, the quenching induced by oleate or dodecyl-LPC, which did not significantly bind to Bid (cf. Figure 1d), was very similar in the absence or presence of Bid (data not shown). This ensured that the modified quenching of ADIFAB observed in the presence of Bid was caused by a shift in binding equilibrium due to competition, a phenomenon that could be exploited to indirectly deduce binding parameters for Bid.

To quantitatively evaluate the affinity of Bid for a lysolipid, we analysed ADIFAB quenching in the presence of (t)Bid by computing the levels of free lysolipid with the mass action equilibrium and $K_{d}$ values previously obtained wth ADIFAB alone. When Bid had more affinity than ADIFAB for a given lysolipid, the Stern-Volmer plots of quenching fitted a flatter slope than with $A D I F A B$ alone (Figure $2 \mathrm{a}$, right), and the change in slope was proportional to the strength of lysolipid association with (t)Bid. The slope change was then used to derive an approximate $K_{d}$ value of (t)Bid for the lysolipid, which was implemented by iterative simulations until a best fit to the experimental data was obtained. The $K_{\mathrm{d}}$ values computed in this way matched those independently obtained by the measurements of Bid intrinsic fluorescence (Figure 1d), which further validated our procedure.

With this procedure we determined that Bid had greater affinity for LPG than LPA, the $K_{\mathrm{d}}$ values for their oleyl analogues being 0.09 and $0.52 \mu \mathrm{M}$, respectively. We also found that caspase cleavage substantially increased the affinity of the protein for MCL (the estimated $K_{d}$ value decreased from 2.90 to $0.69 \mu \mathrm{M}$, Figure $2 \mathrm{a}$ ), while having little effect on the binding to LPA and other lysolipids (not shown). Our results indicating that caspase-cleaved Bid exhibited an increased affinity for $\mathrm{MCL}$ in solution were consistent with the previous report of a preferential association of tBid to liposomes containing $\mathrm{CL}^{8}$

\section{Binding to lysocardiolipins induces Bid aggregates}

The binding studies reported above required micromolar concentrations of Bid, which are higher than those usually present in mammalian cells. To evaluate Bid binding to lipids at physiologically relevant concentrations (cf. Lutter et al.; ${ }^{8}$ Kuwana et al. ${ }^{13}$ ), we used another approach based upon the principle that tight binding to lipids could induce changes in the electrophoretic mobility of the protein (gel shifts) under nondenaturing conditions, as previously reported for some lipid-binding proteins. ${ }^{28}$ Indeed, the electrophoretic migration of f.l. Bid and tBid was significantly retarded by prior incubation with selected lipids or detergents like Tween-20, producing diffuse aggregates that apparently migrated as oligomeric forms - a property shared with Bax, ${ }^{7,29}$ albeit with different specificity (Y Nakao, Y Chen and M Degli Esposti, unpublished data). To systematically test the lipid-induced gel 


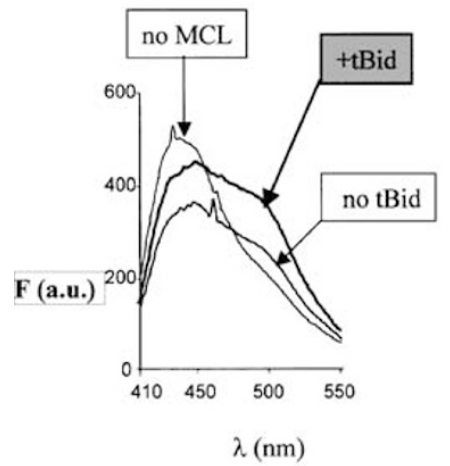

b

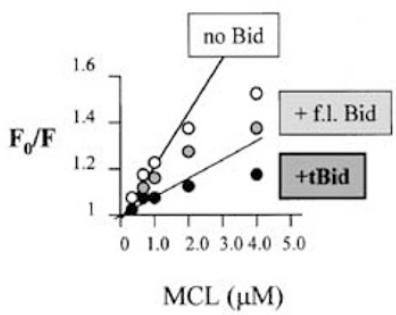

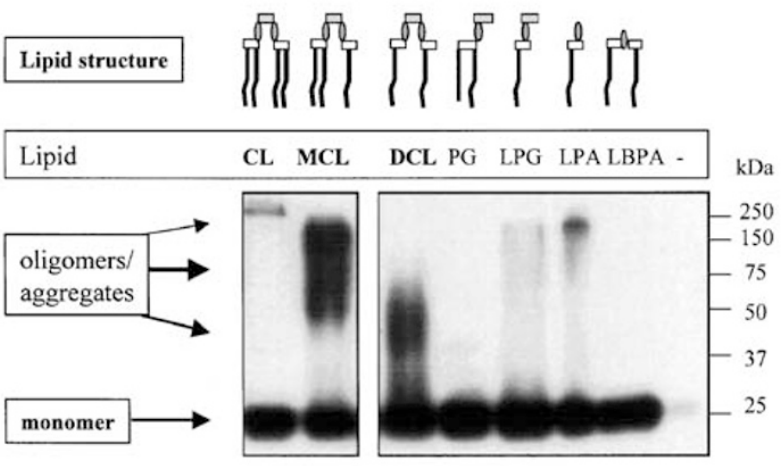

C

PAGE-Western

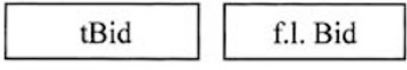

$\operatorname{DCL}_{(\mu \mathrm{M})}$

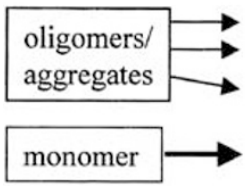

$\begin{array}{llllllllllll}0 & 1 & 2 & 5 & 9 & 18 & 0 & 1 & 2 & 5 & 9 & 18\end{array}$

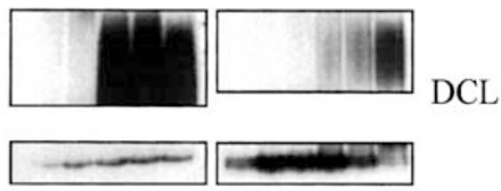

d

$\operatorname{MCL}(\mu \mathrm{M})$

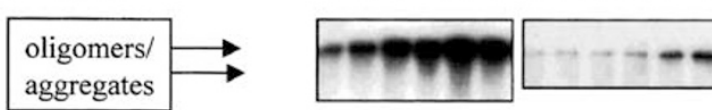

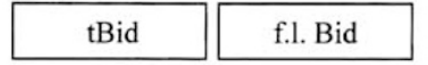

$\begin{array}{llllllllllll}0 & .2 & .5 & 1 & 5 & 9 & 0 & .2 & .5 & 1 & 5 & 9\end{array}$

e

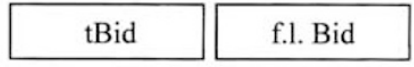

$\mathrm{CL}(\mu \mathrm{M})$

$\begin{array}{llllllllll}0 & .5 & 1 & 5 & 9 & 0 & .5 & 1 & 5 & 9\end{array}$
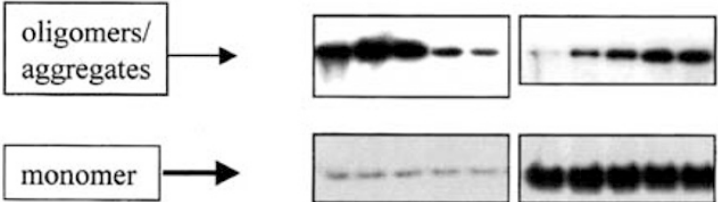

CL

Figure 2 Binding of Bid to lysolipids in solution. (a) Binding measurements in solution were obtained following the fluorescence changes of ADIFAB, ${ }^{26}$ as described in the Experimental procedures. The left panel shows fluorescence spectra of the ADIFAB probe before (no MCL) and after $2 \mu \mathrm{M} \mathrm{MCL}$ in the absence (bottom spectrum no tBid) and presence of $0.2 \mu \mathrm{M}$ caspase 8-cleaved Bid (middle spectrum, + tBid). The spectrum with tBid alone was intermediate between that of ADIFAB alone and that with both tBid and MCL, and is not shown for the sake of clarity. The right panel shows the Stern-Volmer plots of fluorescence quenching used to compute the affinity for MCL: empty circles, no Bid; grey circles, with f.l. Bid; filled circles, with tBid. $F_{0}$ and $F$ have the same meaning as in Figure $1 \mathrm{~d}$. Note that the slope in the linear interpolation of the data points (thin lines) is proportional to the association constant, that is, the inverse of the $K_{\mathrm{d}}$ for the ligand quencher, ${ }^{42}$ and this association is higher for f.I. Bid than caspase-cleaved Bid. Deviation form linearity in the Stern-Volmer plot is indicative of static quenching as reported previously. ${ }^{42}$ (See text for further details.) (b) Interaction of Bid with cardiolipin metabolites was evaluated by PAGE-Western (cf. Arnold et al. ${ }^{28}$ ) after incubating $4 \mathrm{nM}$ f.l. Bid with $20 \mu \mathrm{M}$ of each lipid (from ethanolic stock solutions) for 20 min in assay buffer as in cell-free assays. MCL was prepared from the same CL sample used in the experiment using the in-house procedure described in the Experimental procedures. After separation, the protein was blotted and immunodetected as described. ${ }^{9}$ Data are representative of $n=5$ separate experiments. (c) PAGE-Western profile of the concentration dependence of oligomers/aggregates produced by DCL on $8 \mathrm{nM}$ caspase-cleaved Bid (tBid) (left) and $4 \mathrm{nM}$ f.l. Bid (right). Data were extracted from the same blot maintaining identical migration distances (cf. part (b)) to provide a congruent comparison of the different aggregation and immunodetection effects of the various lipids on Bid proteins. (d) PAGE-Western profile of the concentration dependence of oligomers/aggregates produced by MCL (from Avanti Lipids, cf. part (a)) on tBid and f.I. Bid. (e) PAGE-Western profile of the concentration dependence of oligomers/aggregates produced by $\mathrm{CL}$ (from Sigma) on tBid and f.I. Bid. Note that identical conditions were used in (c) (right), (d) and (e), whereas the tBid blot in (c) (left) was taken with shorter exposure to limit signal saturation of the aggregates/oligomer versus the monomer.

shifts, we refined a protocol of polyacrylamide gel electrophoresis without SDS followed by standard Western blotting (PAGE-Western). The PAGE-Western profile of Bid presented remarkable differences with various lipids related to $\mathrm{CL}$, as shown in Figure $2 \mathrm{~b}$. MCL produced extensive retardation of Bid migration, forming aggregates that appeared to run as diffuse oligomers of the protein. Similar oligomers/aggregates were observed in the presence of dilysocardiolipin (DCL) and, to a lesser extent, cardiolipin and LPA too (Figure 2b). On the other hand, PG and lyso-bisphosphatidic acid (LBPA) - another dimeric lipid which, contrary to $\mathrm{CL}$, is restricted to lysosomes - did not induce comparable changes in Bid migration (Figure 2b), clearly indicating that Bid had a unique capacity to interact and aggregate with some CL metabolites. Indeed, PAGE-Western analysis applied to Bax showed no comparable lipid- 
dependent changes, but strong oligomerisation with certain detergents (cf. Hsu and Youle ${ }^{29}$ ).

Caspase 8-cleaved Bid showed an enhanced propensity to aggregate than f.I. Bid, resulting in being partially oligomeric in the absence of detergents or exogenous lipids (cf. Figure $2 \mathrm{~d}, \mathrm{e})$. Binding of $\mathrm{tBid}$ to $\mathrm{DCL}$ or $\mathrm{MCL}$ produced stable aggregates that migrated much slower than monomeric tBid and could correspond to oligomeric complexes or protein-lipid aggregates. These aggregates, contrary to those induced by detergents like Tween-20, remained resistant to both heat and SDS (Figure 2c,d and data not shown; see also Grinberg et $\mathrm{al}^{10}{ }^{10}$ ). Evaluation of the concentration dependence of aggregate formation in PAGE-Western confirmed the evidence previously obtained with ADIFAB (Figure 2a) that caspase cleavage increased the affinity of Bid for $\mathrm{CL}$ metabolites (Figure 2c-e). PAGE-Western analysis could also be applied to CL (Figure 2e), which appeared to induce oligomers/aggregates of (t)Bid with reduced efficiency and a different concentration dependence than either MCL or DCL (Figure 2c-e).

\section{MCL promotes Bid association with mitochondria}

Next, we extended the observations of lipid binding in solution to the interaction of Bid with lipid membranes in two model systems: (1) cell-free assay with isolated liver mitochondria, recombinant Bid and exogenous lipids (cf. Degli Esposti et $a .^{9}{ }^{9}$;. (2) artificial membranes constituted by different phospholipids, including those extracted from mitochondria (cf. Kuwana et al. ${ }^{13}$ ).

Figure 3 Preferential interaction of Bid with MCL for membrane association. (a) The distribution of f.l. Bid ( $4 \mathrm{nM}$ ) before (upper panel) and after (lower panel) cleavage with caspase-8 (tBid) was measured in the pellet of cell-free assays after incubation with $20 \mu \mathrm{M}$ of each lipid or an equivalent volume of solvent (none). Note the increased levels of both f.l. Bid and tBid with MCL. (b) Data were obtained with $8 \mathrm{nM}$ tBid incubated with a 10 -fold lower concentration of lipids than in (a) to evaluate the different effect of $\mathrm{MCL}$ and $\mathrm{CL}$; porin reblot (bottom panel) documents pellet loading. (c) Part i shows the release of cytochrome $c$ (cyt $c$ ) in the supernatant of mitochondria incubated with lipids alone (upper panel) or together with caspase 8-cleaved Bid (+ tBid, lower panel). The control (none) had the same volume of ethanol $(0.5 \%)$ as the samples containing lipids (the concentration of which was held at $2 \mu \mathrm{M}$ as in (b)). Note the negligible effect of $\mathrm{LPG}$ and MCL alone in comparison with the increased release of cytochrome $c$ induced by either the positive control of $50 \mu \mathrm{M}$ digitonin (upper panel) or by tBid, particularly in the presence of $L P G$ and MCL (lower panel). Results obtained in the presence of $P G, C L$ and $D C L$ alone were superimposable to the controls (not shown). Part ii shows the distribution of tBid in the pellet of the same supernatant samples as in part $\mathrm{i}$, with the bottom panel presenting the porin reblot to document sample loading. Evaluation of cytochrome $c$ in the same mitochondrial pellets could not be performed accurately due to overexposure of the blots. Results were confirmed in $n=3$ replicates. (d) Binding of Bid to lipid membranes was evaluated using liposomes $(0.1 \mathrm{mg} / \mathrm{ml})$ prepared by ethanol injection with a mixture of PC : PI : PS ( $50: 30: 20$ by weight), supplemented when indicated with $5 \%$ molar fraction of $C L$ or $M C L$, individually or in a 1:1 combination. Note that in the latter case (lane 3) the total molar fraction of $C L$ and $M C L$ combined was the same as that with either lipid alone. In the right panel, liposomes were obtained from a lipid extract of mouse liver mitochondria, in the absence (lane 6) or presence (lane 7 ) of exogenous $\mathrm{MCL}$ (corresponding to about a $10 \% \mathrm{~mol}$ fraction in the sample). The bottom panels show the total amount of Bid added (input) before separation of the liposomal pellets by centrifugation at $18000 \times g$ after the addition of $10 \mathrm{mM} \mathrm{CaCl}$. Addition of $\mathrm{CaCl}_{2}$ without centrifugation did not significantly affect membrane binding of Bid. However, calcium appeared to partly enhance the immunodetection of Bid in the pellet of liposomes containing $\mathrm{CL}$ or MCL with respect to the input liposomes (without calcium), accounting for some apparent quantitative discrepancies in the blots.
In the first model, we used the same conditions as in PAGE-Western studies (Figure $2 \mathrm{~b}$ ) and investigated whether exogenous CL metabolites could modify Bid association to mitochondria after their separation by centrifugation. Although CL promoted tBid binding to membranes, in agreement with previous reports, ${ }^{8,13} \mathrm{MCL}$ was more effective than $\mathrm{CL}$ in a wide range of concentrations (Figure $3 a$ and $b$ ). Interestingly, the closely related DCL did not significantly increase the association of either f.I. Bid or tBid to mitochondria (Figure 3a). Considering this and also the negligible amount of $\mathrm{DCL}$ present in mitochondria (see MS data below), we focused subsequent studies on MCL rather than DCL.

We then decreased the concentration of exogenous lipids to levels that did not significantly affect the basal release of cytochrome $c$ in the supernatant (also compared to solvent controls, Figure 3ci), but could influence the association of caspase-cleaved Bid to the mitochondrial pellet (Figure 3b, cii). Under these conditions, the added lipids were mostly incorporated into the mitochondrial membrane, thereby minimising micelle formation in solution. The effect of $\mathrm{MCL}$ was unique, in that it concomitantly increased tBid association to mitochondria and the release of cytochrome $c$ (Figure $3 c$ ). LPG also increased the cytochrome $c$ releasing capacity of tBid, but not its association with mitochondria (Figure $3 \mathrm{c}$ ),

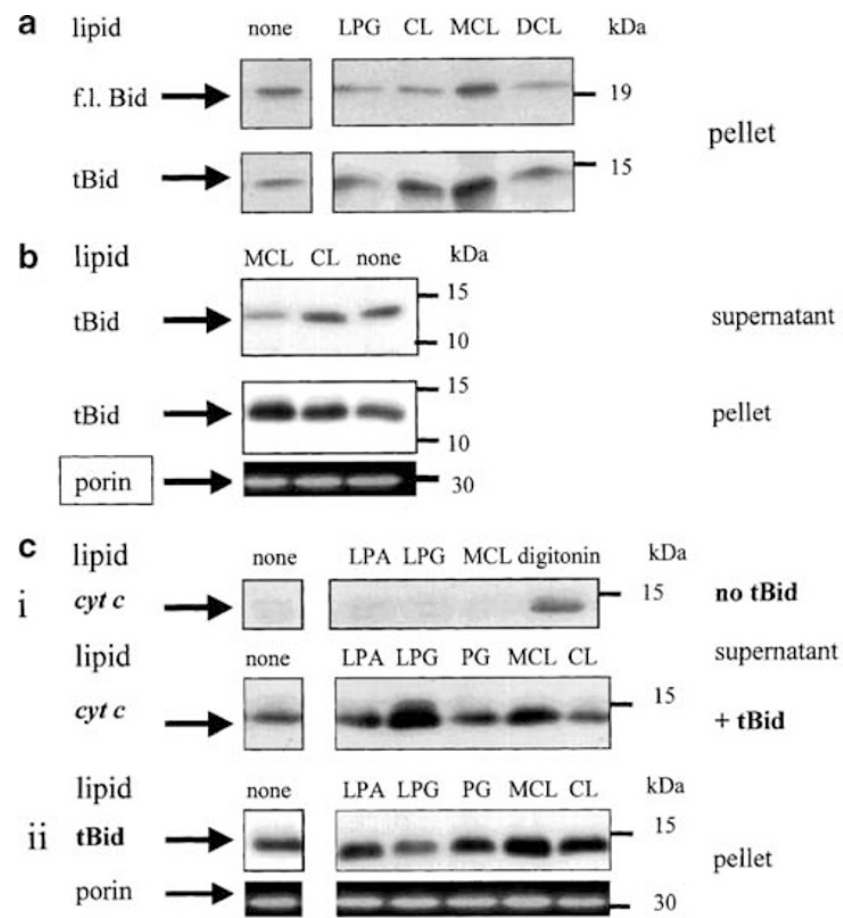

d liposome lipid mix of PC:PS:PI mitochondrial

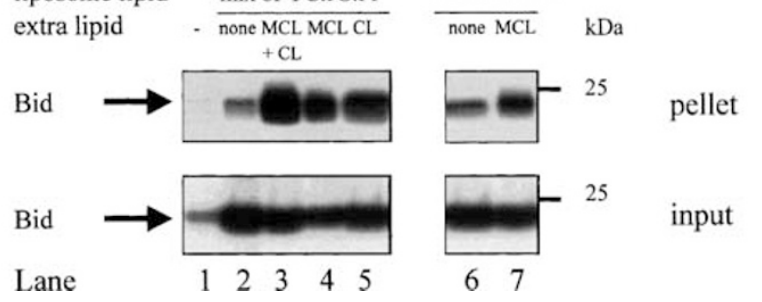


which was verified by increased levels of the protein in the supernatant (not shown). Conversely, MCL increased the association f.I. Bid to mitochondria (Figure 3a), without major changes in cytochrome $c$ release (data not shown). Hence, the in vitro pattern of $\mathrm{MCL}$ effects on exogenous Bid was distinctive, representing a potential blueprint for apoptosismediated changes in endogenous Bid.

In the second model, we consistently found that inclusion of MCL in the membrane composition enhanced Bid association to liposomes (of either small or large size). This was particularly evident when the lipid composition of the liposomes matched that of the mitochondrial outer membrane (Figure 3d). The presence of cardiolipin in the membrane also increased the association of f.I. Bid (Figure 3d, left) and tBid (not shown, cf. Lutter et al. ${ }^{8}$ ). However, equimolar levels of $\mathrm{MCL}$ and $\mathrm{CL}$ increased Bid binding to the liposome membrane much more than either lipid alone (Figure 3d, lane 3). Moreover, the addition of exogenous MCL to liposomes formed with mitochondrial lipid extracts - thus containing natural $C L$ - enhanced Bid binding to membranes (right panel in Figure 3d). Instead, the addition of exogenous CL had little effect on the association of Bid with these liposomes (results not shown, cf. Degli Esposti et $a^{\beta}$ ).

In summary, the results obtained with artificial lipid membranes were consistent with those obtained with mitochondria in confirming that MCL was more effective than $C L$ in promoting membrane association of the protein. Regarding the quantitative evaluation of immunoblots, some concern arose from the observation that MCL enhanced Bid immunodetection. Nevertheless, this effect was shared with lysolipids like LPG that did not promote Bid relocation to mitochondria (Figure $3 a$ and cii), and thus could be ascribed to conformational changes arising from surfactant interaction with the protein, as also indicated by intrinsic fluorescence data (Figure 1d). Moreover, lipid-induced changes in immunodetection were fundamentally accounted for by comparison with the blots of the samples before separation of the liposomes (input data, bottom panel in Figure $3 d$ ) and some enhancement produced by calcium treatment of $\mathrm{CL}$-containing liposomes (not shown).

Figure 4 Analysis of mitochondrial lipids and endogenous Bid in apoptotic liver. (a) ES-MS spectra were obtained in lipid extracts of mitochondria isolated from control and Jo-2-treated livers; they show the multiple species of liver cardiolipin, including the prominent $[18: 1,(18: 2) 2,22: 6+3 \mathrm{Na}](\mathrm{m} / \mathrm{z}=1566)$ and $[(18: 2) 3,20: 4+3 \mathrm{Na}](\mathrm{m} / \mathrm{z}=1542)$. Of note, commercial $\mathrm{CL}$ predominantly contained the species $[(18: 2) 4+3 \mathrm{Na}](\mathrm{m} / \mathrm{z}=1516)$ (cf. Figure $1 \mathrm{c})$. (b) The main panel shows the relative ratio between major $M C L$ and parent $C L$ species (white histograms, $\quad n=4)$ compared with that between [(18:2)3] $\mathrm{MCL}$ and $[(18: 2) 3,20: 4] \mathrm{CL}$ (grey histograms). The inset shows the cytochrome $c$ content of the same mitochondria used for MS analysis. (c) Western blots of key mitochondrial proteins were undertaken along the progression of Jo-2 treatment using $20 \mu \mathrm{g}$ of protein per lane. Bid was blotted overnight at $4^{\circ} \mathrm{C}$ with a mixture of the R\&D and C-20 (SantaCruz) antibody that was optimised for detecting endogenous tBid. The middle panel shows the content of cytochrome $c$ in the same mitochondrial samples, while the bottom panel presents data of $n=3$ measurements of caspase activity in the corresponding cytosolic extracts (fraction $\mathrm{S} 10)$, which were normalised to the activity of control samples as in Li et al..$^{32}$ Note that after $2 \mathrm{~h}$ of Fas activation, there was only a small increase in caspase activity of ex vivo treated liver (not shown). The intensity changes in the f.l. Bid band and in the 117 band (asterisk) were detected in five separate experiments and also with other antibodies (cf. Li et al..$^{32}$ and Sarig et al. ${ }^{34}$ ).

\section{Changes in mitochondrial lipids during Fas-induced apoptosis}

To investigate the significance of the above in vitro results to mitochondria-dependent apoptosis in vivo, we carried out sensitive measurements of CL metabolites before and after apoptosis induction; previously, MCL had been evaluated only in metabolic studies. ${ }^{21,30}$. By applying electrospray (ES) MS, we were able to measure the basal levels of $M C L$ in mitochondrial lipid extracts from liver (Figure 4a) and human cells (M Sorice, A Cirella, I Cristea, G Valesini and M Degli

a

ES-MS spectra

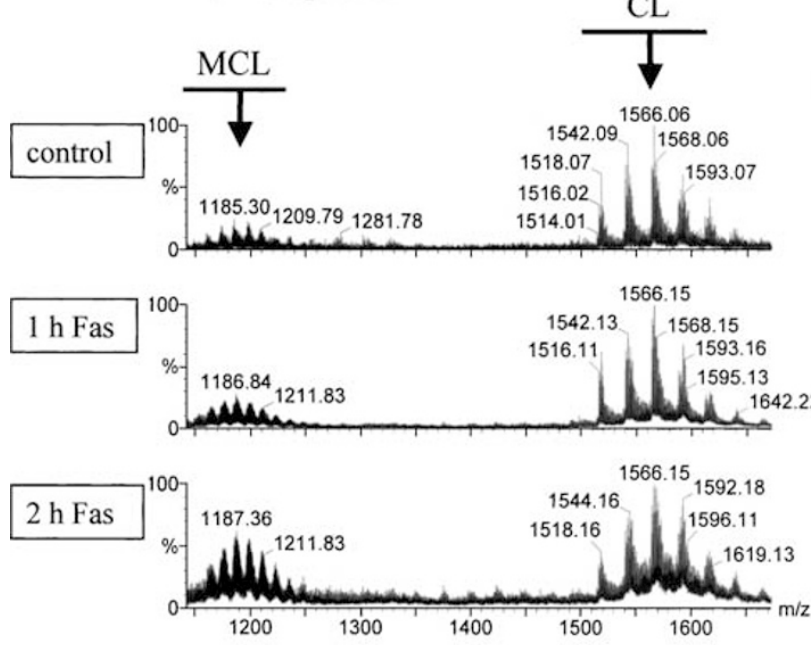

b Ratio of MCL vs. CL

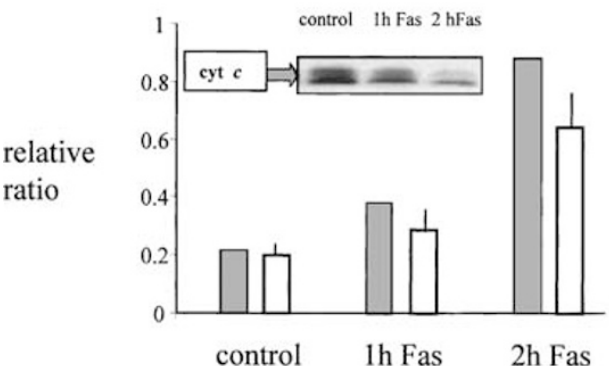

$\operatorname{species}(\mathrm{m} / \mathrm{z})$

C

Fas $\quad-1 \mathrm{~h} 3 \mathrm{~h} 4 \mathrm{~h} \quad \mathrm{kDa}$

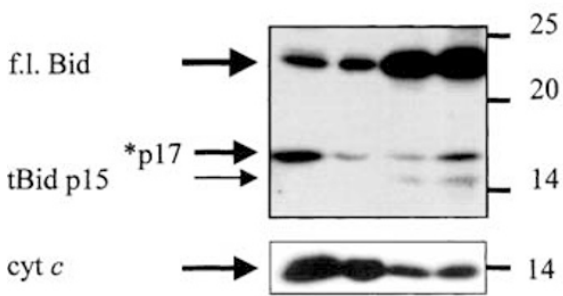

mitochondria

$\square 1186 / 1542$

$\square$ average

relative

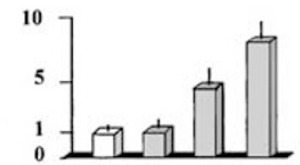

cytosol (S10) caspase activity

Fas
- 1h 3h 4h 
Esposti, manuscript in preparation). In liver, the dominant MCL species were identified by the peaks around $\mathrm{m} / \mathrm{z}$ 1200 (Figure 4), which corresponded to salts of tri-linoleoyl $(18: 2)$ and di-linoleoyl, palmenyl $(16: 1) \mathrm{MCL}$, related to the predominant species of mammalian cardiolipin. ${ }^{18,22}$ Despite the sensitivity of our MS analysis, we did not detect peaks of significant intensity that could be unambiguously assigned to DCL species (data not shown), consistent with the notion that DCL was not efficiently metabolised in isolated liver mitochondria. ${ }^{22,30}$ The acquired capacity of analytically measured MCL was then applied to discern changes in cardiolipin homeostasis induced by apoptosis.

We focused here on an established model of apoptosis in which Bid has been shown to be important for releasing mitochondrial apoptogenic factors, namely activation of Fas by the Jo-2 agonist antibody in mouse liver. ${ }^{31,32}$ Similar results were obtained in type II cells in culture, for example, U937 (M Sorice, A Cirella, I Cristea, G Valesini and M Degli Esposti, manuscript in preparation). Fas-induced apoptosis progressively decreased the content of mitochondrial cardiolipin in mouse liver as in human cells (cf. Matsko et al. $^{18}$ ), and this decrease corresponded to an increase in various MCL species (Figure $4 a, b$ ). To obtain a quantitative evaluation of the transition of $\mathrm{CL}$ into MCL during apoptosis, we computed the relative ratio of major MCL species with their $\mathrm{CL}$ precursors, as shown in Figure 4b. On average, this ratio increased approximately two-fold after $1 \mathrm{~h}$ of Fas activation in the liver, and then nearly four-fold after $2 \mathrm{~h}$ of Fas activation (Figure 4b, main panel). However, some CL species were comparatively more degraded than others, suggesting some specificity presumably related to $\mathrm{CL}$ remodelling (cf. Ma et al. ${ }^{21}$ ).

Progression of Fas-induced apoptosis was accompanied by some changes in other mitochondrial lipids, especially after $2 \mathrm{~h}$ of Fas activation. These changes included an increase in lysolipids that could bind to Bid, like LPA and LPG (data not shown), but quantitatively they did not match the early increase in MCL, and apparently did not derive from a complementary decrease in parent mitochondrial lipids. We also detected previously unidentified peaks in the mass region between 950 and 1150 that could derive from degradation products of MCL or $P G$, but did not match the expected mass of DCL species (I Cristea and M Degli Esposti, unpublished observations). The uncertain molecular assignment of these peaks has so far prevented correlating them with specific degradative processes linked to death signalling (a dedicated study is under way to clarify this). In any case, we could not relate the observed changes in MCL and other lipid species to an increased activity of mitochondrial phospholipase A2 (PLA2), since no significant increase in this activity (either calcium dependent or independent) was detected in mitochondria from livers treated with Jo-2 for $1-2 \mathrm{~h}$ (results not shown). However, the accumulation of MCL concomitant with $\mathrm{CL}$ decline paralleled the loss of mitochondrial cytochrome $c$ (Figure $4 \mathrm{~b}$, inset), suggesting a relation between the integrity of mitochondrial membranes and their altered lipid composition. ${ }^{12,20}$

\section{Changes in endogenous Bid of mitochondria during Fas-induced apoptosis}

We previously reported that mitochondria of primary tissues contained a complement of endogenous Bid producing multiple reacting bands in immunoblots. ${ }^{9}$ In the light of the above results, we reasoned that perhaps it would be possible to establish a relation between measurable changes in the profile of mitochondrial Bid forms and the changes in mitochondrial lipids induced by Fas-mediated apoptosis (Figure 4a). A primary concern was to detect the relocation of endogenous tBid to mitochondria that is considered crucial in Fas-mediated apoptosis. ${ }^{1-4,31,32}$ Even if an initial cleavage of cytosolic f.I. Bid was apparent after $1 \mathrm{~h}$ of Fas activation (cf. Gross et al.; ${ }^{4}$ Yin et al. ${ }^{31}$ ), tBid association with mitochondria could be clearly detected only at subsequent times, when evident increase in caspase activity could also be measured (typically after $3 \mathrm{~h}$ of Fas activation under our conditions, Figure 4c). On the other hand, we did find that Fas activation changed the immunodetectable forms of mitochondrial Bid, with a consistent increase in the major band of f.l. Bid (Figure 4c, cf.Degli Esposti et al.; ${ }^{9}$ Tafani et al.;3 Sarig et $\left.a .^{34}\right)$. Hence, Fas-mediated apoptosis induced an increase of both f.I. Bid and tBid in mitochondria (Figure 4c) with concomitant release of cytochrome $c$ (Figure $4 \mathrm{~b}$, inset, and Figure 4c), a pattern resembling that obtained in vitro with exogenous MCL (Figure $3 a-c$ ) and suggesting a redistribution of endogenous Bid between intracellular organelles.

Diverse antibodies detected also a Bid-reacting band that broadly migrated between f.l. Bid and p15 tBid (labelled p17 in Figure $4 \mathrm{c}$ ). This band, which has been seen before, ${ }^{32,34}$ did not arise from caspase cleavage of Bid, since its intensity was unaffected by $z-V A D$ and was not increased by ectopic expression of tBid. ${ }^{10,34}$ In fact, the p17 band decreased in mitochondria during the progression of Fas-induced apoptosis (Figure 4c), suggesting that it originated from aberrant adducts of Bid with mitochondrial lipids and SDS. Following this interpretation, the progressive decrease of p17 Bid during Fas activation (Figure 4c) may also reflect a decrease in mitochondrial $\mathrm{CL}$ species that are rapidly degraded during apoptosis, for example, tri-linoleoyl, arachidoyl-CL $(\mathrm{m} /$ $z=1542$, Figure $4 a, b)$, and thus become a potential expression of the altered lipid composition of mitochondria during apoptosis.

\section{Discussion}

It is increasingly appreciated that the mechanism of proapoptotic proteins of the Bcl-2 family like Bid involves crucial interactions with membrane lipids that favour membrane permeabilisation. ${ }^{1,17}$ Herein we report that Bid displays binding specificity for lysolipids related to cardiolipin, especially MCL, and that caspase cleavage of the protein increases its affinity for the same lipids (Figure 2). Moreover, we have found that Fas-mediated apoptosis in liver, which requires Bid for its amplification via the release of mitochondrial apoptogens, ${ }^{31}$ is associated with progressive accumulation of MCL derived from cardiolipin degradation (Figure $4 a, b$ ). This is parallel to a gradual relocation of f.I. Bid and tBid to mitochondria (Figure 4c). Since MCL promotes the associa- 
tion of Bid with membranes independently of their content of $\mathrm{CL}$ (Figure 3), we conclude that $\mathrm{MCL}$ facilitates (t)Bid relocation to mitochondria during apoptosis, especially when $\mathrm{CL}$ is degraded. This would solve the apparent paradox between the evidence indicating a requirement for $C L$ in the proapoptotic action of Bid and $\mathrm{Bax}^{1,8,13,14}$ and the evidence for a decrease in CL during cell death. ${ }^{18-20}$

Cardiolipin degradation with simultaneous MCL accumulation suggests that apoptosis signalling induces alteration in the remodelling cycle of mitochondrial membranes. ${ }^{11,16}$ Of note, MCL resides in both membranes of mitochondria and is involved in remodelling nascent cardiolipin into its mature species. $^{21,22,30}$ Apoptosis mediated by death receptors has been shown to enhance the remodelling of cellular lipids, ${ }^{35}$ in part due to early activation of phosphatidylcholine-specific phospholipase $\mathrm{C}\left(\mathrm{PC}-\mathrm{PLC}^{36}\right)$. For instance, the increase of PC-PLC activity could impact on the remodelling cycle of cardiolipin by depleting PC, which is the preferred donor for MCL re-acylation. ${ }^{30}$ The consequent imbalance in remodelling reactions would decrease the levels of mature $C L$ species and increase those of precursor MCL species, as observed during Fas-induced apoptosis in liver (Figure 4a) and type II cells like U937 (M Sorice, A Cirella, I Cristea, G Valesini and M Degli Esposti, manuscript in preparation). CL transformation into MCL may not derive from enhanced activity of mitochondrial PLA2, since this activity does not increase within $2 \mathrm{~h}$ of Fas-induced apoptosis, and would be potently inhibited by MCL. ${ }^{37}$ However, death receptor activation of other phospholipases has been reported $^{36,38}$ and could dynamically contribute to the observed changes in mitochondrial lipids. In this respect, it would be interesting to determine whether lysosomal lipases - the enzymes normally responsible for recycling cardiolipin ${ }^{20}$ - are either activated or released from the lumen of lysosomes/endosomes and contribute to the degradation of mitochondrial lipids during apoptosis. Although we did not find evidence for an increased contamination of liver mitochondria by lysosomal components early after Fas activation, it remains possible that the new lipid peaks we identified in apoptotic mitochondria could derive from processes mediated by lysosomal or endosomal factors. Studies are under way to verify this possibility.

In mouse liver, MCL accumulation is observed in parallel to mitochondrial relocation of (t)Bid and cytochrome $c$ release (Figure 4), a situation that can be modelled in vitro by the addition of low concentrations of exogenous MCL to mitochondria and tBid (Figure 3). While this does not imply a direct participation of Bid in the metabolic reactions leading to $\mathrm{CL}$ degradation, it strongly indicates a connection between its mitochondrial association and apoptotic changes in mitochondrial lipids, including the elevation of MCL that occurs before ouvert caspase activation (Figure 4). Hence, MCL may play a crucial role in connecting metabolic reactions of mitochondrial lipids with tBid binding to the $\mathrm{OM}$ and subsequent loss of membrane integrity. That cardiolipin decreases in many pathways of apoptosis ${ }^{18-20}$ can now be reconciled with its involvement in the apoptogenic action of $\mathrm{tBid}^{1,8,13,14}$ by considering that $\mathrm{MCL}$, a direct by-product of cardiolipin degradation, promotes tBid interaction with membranes.

To conclude, we would like to consider the peculiar molecular properties of MCL that may be relevant to its new proposed role. $\mathrm{MCL}$ is unique among membrane lipids because it combines the properties of a di-acyl phospholipid forming bilayers with those of a lysolipid like LPA, promoting bilayer tension and discontinuity. ${ }^{12}$ Given the "natural selection' of Bid for binding to bacterial LPA (cf. Figure 1) and Bid capacity to form stable aggregates with MCL (Figure 2), it is conceivable to propose that Bid or tBid form aggregates in local areas of the membrane that are enriched in $\mathrm{MCL}$. The consequent segregation of MCL molecules would produce 'lipid domains' in the $\mathrm{OM} ;{ }^{1,13,17}$ the different physical properties of these domains could then 'prime' the membrane surface for the clustering of Bak or Bax and subsequent formation of protein-lipid pores that break membrane integrity (cf. Newmeyer and Ferguson. ${ }^{1}$; Hardwick and Polster ${ }^{17}$ ). The next challenge will be to isolate and characterise these putative lipid domains in mitochondria.

\section{Experimental procedures}

\section{Antibodies and proteins}

To detect native Bid we used diverse antibodies (R\&D Systems goat AF860, Santa Cruz C-20, and rabbit $224 \mathrm{~A}^{39}$ ). Proteins were separated with standard SDS-PAGE or with seminative PAGE, that is, PAGE-Western (without SDS in gel and buffers as in Arnold et al. ${ }^{28}$ ). The gels routinely contained $15 \%$ acrylamide and were blotted as described previously. ${ }^{9}$ Blots were visualised by chemiluminescence. Recombinant proteins of mouse Bid and caspase 8-cleaved Bid were obtained from R\&D Systems, ${ }^{9}$ or with a His-tag at the $\mathrm{N}$ terminus. $^{7}$

\section{Lipids and other materials}

Lipids and lysolipids were obtained from either Avanti Polar Lipids or Sigma; their purity was checked by thin layer chromatography and, in some cases, also by MS analysis (cf. Figure 1). Owing to the specific effects of MCL, we prepared this lipid also in-house using the procedure below, modified from that described in Schlame and Rustow. ${ }^{30}$ A $40 \mu$ l volume of an ethanolic solution of commercial cardiolipin $(0.2 \mathrm{mg}$ in total) was mixed with $70 \mu$ l of digestion buffer ( $50 \mathrm{mM}$ Tris-Cl, $\mathrm{pH} 7.4$, containing $2 \mathrm{mM} \mathrm{CaCl} 2$ ) and $33 \mu \mathrm{l}$ of PLA2 from porcine pancreas $(3 \mathrm{mg} / \mathrm{ml})$ and then supplemented with $0.9 \mathrm{ml}$ of pure ethanol. The mixture was incubated on a shaker in the dark for $70 \mathrm{~min}$ at room temperature and then centrifuged at 12000 RPM for 10 min to remove protein and other insoluble material. The cleared supernatant was subsequently mixed with $0.2 \mathrm{ml}$ of a solution containing $6 \mathrm{mg} / \mathrm{ml}$ of defatted BSA (to remove fatty acids and other contaminants) and $20 \mathrm{mM}$ EGTA (to remove calcium and block phospholipase); after $10 \mathrm{~min}$ incubation, BSA and residual PLA2 were removed by ultrafiltration through a YM30 filter (Amicon) and the filtrate supplemented with chloroform for MCL extraction and thin layer chromatography.

MCL and other lipids were dissolved in chloroform or ethanol in concentrated stock solutions (their concentration was evaluated by phosphorus content with the Malachite green assay), and then diluted to approximately $1 \mathrm{mM}$ in ethanol before addition to various assays. Liposomes were 
prepared by multiple size fractionation (large unilamellar vesicles, cf. Zha et al. ${ }^{15}$ ) or ethanol dilution (cf. Degli Esposti et al. $^{9}$ ); in the latter case they formed predominantly small unilamellar vesicles of size similar to that obtained by sonication.

\section{Fractionation and Fas activation of liver}

Treatment of mouse livers ex vivo with Jo-2 (BD-Pharmingen, $10-20 \mu \mathrm{g} / \mathrm{ml}$ ) and subcellular fractionation were carried out as described in Degli Esposti et al. ${ }^{9}$. Mitochondria were resuspended in assay buffer $(20 \mathrm{mM} \mathrm{K}$-HEPES, $0.12 \mathrm{M}$ mannitol, $0.08 \mathrm{M} \mathrm{KCl}, 1 \mathrm{mM}$ EDTA, $\mathrm{pH}$ 7.4). The purity of mitochondria was verified by enzyme markers, Western blotting (cf. Degli Esposti et al. ${ }^{9}$ ) and MS analysis of lipids like LBPA. Protein concentration was determined with the BioRad Bradford assay in the presence of Triton X-100.

\section{Mass spectroscopy of lipids}

Extraction of mitochondrial lipids was undertaken as follows. Mitochondrial protein $(5 \mathrm{mg})$ was pelleted by centrifugation and treated with $1 \mathrm{ml}$ methanol and subsequently with $2 \mathrm{ml}$ of chloroform. After vigorous mixing for $5 \mathrm{~min}$, samples were further mixed with $0.5 \mathrm{ml}$ of $0.15 \mathrm{M} \mathrm{NaCl}^{18}$ and the organic phase was separated by centrifugation, dried under nitrogen, dissolved in chloroform, and recentrifuged to remove any insoluble material. Nanospray MS data were acquired in positive mode, ${ }^{40}$ on a Q-ToF I instrument with a dual electrospray source (Micromass, Manchester, UK). For quantitative analysis, we used both nonphysiological lipids such as Bis-BODIPY $\mathrm{FL}^{\circledR} \mathrm{C}_{11}$-PC (also used to evaluate phospholipase activity, cf. Hendrickson et al. ${ }^{41}$ ) and endogenous lipids that were found to remain essentially unaltered either after prolonged Fas-induced apoptosis or treatment with phospholipase $\mathrm{A} 2$, for example, sphingomyelin $(\mathrm{m} /$ $z=703.6$ in its protonated form).

\section{Evaluation of lipid binding}

To evaluate the binding of Bid to various lipids, we followed direct and indirect approaches. The direct approach was based on changes in the intrinsic fluorescence of f.I. Bid that were elicited by water-soluble lysolipids. As in the case of other lipid-interacting proteins, ${ }^{24,25,27}$ the binding to selected lipids changed the whole spectrum of fluorescence emission of Bid (with excitation at $270 \mathrm{~nm}$ ) indicative of altered solvent exposure of aromatic residues in the protein. This approach could be applied to both human and mouse f.l. Bid (at 0.4$0.8 \mu \mathrm{M}$ ) but not to $\mathrm{tBid}$, which at these concentrations was multimeric and poorly soluble (cf. Figure 2c). A major indirect approach exploited the fluorescence quenching of the fatty acid-sensitive probe ADIFAB (Molecular Probes, cf. Richieri et al. $\left.^{26}\right)$. This probe also responds to mono-acyl lysolipids and detergents (our own observations), similarly to other fatty acid binding proteins with bound fluorophores. ${ }^{27}$ ADIFAB was dissolved in assay buffer either alone or in the presence of equimolar levels of Bid (or caspase 8-cleaved Bid, tBid). Emission spectra were recorded using excitation wavelength at $390 \mathrm{~nm}$ in the absence of ligands, with solvent alone, and with increasing concentrations of lipid ligands, either below or above their critical micellar concentration (that, when possible, was measured under the same assay conditions by a modification of the lipid transfer protocol with the BODIPY-PC probe, cf. Degli Esposti et al. ${ }^{9}$ ). Quenching measurements were carried out in a final volume of $2 \mathrm{ml}$ at room temperature (22-24C ${ }^{\circ}$ ) using a Perkin-Elmer LS-500 instrument and maximal quenching $\left(F_{\max }\right)$ was empirically obtained in each cuvette after equilibration with a saturating concentration of the strong ligand oleate (cf. Richieri et al. ${ }^{26}$ ). Lipid binding was determined using numerical elaboration of the analysis reported earlier ${ }^{26}$ according to the following equation:

$$
K_{\mathrm{d}}=\left(F-F_{\max }\right)\left(x_{0} / F_{0}-F-y_{0} / F_{0}-F_{\max }\right)
$$

where $F_{0}$ is the fluorescence in the absence of ligand (corrected for solvent, when required), $F_{\max }$ is the residual ADIFAB fluorescence with excess oleate (generally ca. onesixth of $F_{0}$ ), $F$ is the experimental value of fluorescence at the $x_{0}$ concentration of tested ligand, $x_{0}$ is the concentration of the tested ligand, for example, lysolipid added to the cuvette, and $y_{0}$ is the concentration of ADIFAB in the cuvette - usually fixed at $0.2 \mu \mathrm{M}$ (cf. Richieri et al. ${ }^{26}$ ).

By using the above equation, we obtained a mean $K_{d}$ of $0.23 \pm 0.07 \mu \mathrm{M}(n=4)$ for the specific ligand oleate, in fair agreement with the value of $0.28 \mu \mathrm{M}$ reported previously. ${ }^{26}$ Having validated the method, we performed parallel titrations of various lysolipids in the absence and presence of Bid and analysed the concentration dependence of quenching using Stern-Volmer plots and their mathematical interpretation as described earlier. ${ }^{42}$ (See text for further details.)

\section{Other assays}

Cell-free assays were carried out as described ${ }^{9}$ with $1 \mathrm{mg} / \mathrm{ml}$ of mouse liver mitochondria dissolved in assay buffer and supplemented with Bid preparations and lipids as required. Samples were incubated at room temperature for $20 \mathrm{~min}$ with the tested lipids dissolved in ethanol (the final concentration of which never exceeded 1\%), and mitochondria were separated by centrifugation at $10000 \times g$ for $15 \mathrm{~min}$. Equivalent volumes of supernatants and pellets were then analysed with Western blotting. ${ }^{9}$

Caspase activity was measured in fraction $\mathrm{S} 10$ using DEVD-AMC as fluorogenic substrate. ${ }^{9,10}$

Phospholipase activity of mitochondria was assayed following the quenching of the ADIFAB probe by endogenous fatty acids, ${ }^{26}$ by measuring the fluorescence increase of BisBODIPY FL ${ }^{\circledR} \mathrm{C}_{11}-\mathrm{PC},{ }^{41}$ and also by MS analysis of lipids. The PC-PLC activity was measured with a dedicated kit (A-12218 Molecular Probes).

\section{Acknowledgements}

We thank M Smith, J Erler and Y Chen for help in different aspects of this work, and J-C Martinou for the generous gift of Bid samples and support. D Broomhead, R Kluck, D Green, A Gross, M Schlame, G Basanez, G Valesini, S Korsmeyer and R Khosravi-Far are thanked for discussions. This work was sponsored by AICR Grant no. 03-146. 


\section{References}

1. Newmeyer DD and Ferguson-Miller S (2003) Mitochondria: releasing power for life and unleashing the machineries of death. Cell 112: 481-490

2. Wang $X$ (2001) The expanding role of mitochondria in apoptosis. Genes Dev. 15: $2922-2933$

3. Luo X, Budihardjo I, Zou H, Slaughter C and Wang X (1998) Bid, a Bcl2 interacting protein, mediates cytochrome $c$ release from mitochondria in response to activation of cell death receptors. Cell 94: 481-490

4. Gross A, Yin XM, Wang K, Wei MC, Jockel J, Milliman C, Erdjument-Bromage $\mathrm{H}$, Tempst $\mathrm{P}$ and Korsmeyer SJ (1999) Caspase cleaved BID targets mitochondria and is required for cytochrome $c$ release while $B C L-X L$ prevents this release but not tumor necrosis factor death. J. Biol. Chem. 274: 1156-1163

5. Degli Esposti M (2002) The roles of Bid. Apoptosis 7: 433-440

6. Wei MC, Zong WX, Cheng EH, Lindsten T, Panoutsakopoulou V, Ross AJ, Roth KA, MacGregor GR, Thompson CB and Korsmeyer SJ (2001) Proapoptotic $B A X$ and $B A K$, a requisite gateway to mitochondrial dysfunction and death. Science 292: 62462-624626

7. Kudla G, Montessuit S, Eskes R, Berrier C, Martinou J-C, Ghazi A and Antonsson B (2000) The destabilisation of lipid membranes induced by the C-terminal fragment of caspase 8-cleaved Bid is inhibited by the N-terminal fragment. J. Biol. Chem. 275: 22713-22718

8. Lutter M, Fang M, Luo X, Nishijima M, Xie X and Wang X (2000) Cardiolipin provides specificity for targeting of tBid to mitochondria. Nat. Cell Biol. 2: $754-756$

9. Degli Esposti M, Erler ET, Hickman JA and Dive C (2001) Bid, a widely expressed pro-apoptotic protein of the Bcl-2 family, displays lipid transfer activity. Mol. Cell Biol. 21: 7268-7276

10. Grinberg M, Sarig R, Zaltsman Y, Frumki D, Grammatikakis N, Reuveny E and Gross A (2002) tBID homooligomerizes in the mitochondrial membrane to induce apoptosis. J. Biol. Chem. 277: 12237-12245

11. Scorrano L, Ashiya M, Buttle K, Weiler S, Oakes SA, Mannella CA and Korsmeyer SJ (2002) A distinct pathway remodels mitochondrial cristae and mobilizes cytochrome $c$ during apoptosis. Dev. Cell 2: 55-67

12. Basanez G, Sharpe JC, Galanis J, Brandt TB, Hardwick JM and Zimmerberg J (2002) Bax-type apoptotic proteins porate pure lipid bilayers through a mechanism sensitive to intrinsic monolayer curvature. J. Biol. Chem. 277: 49360-49365

13. Kuwana T, Mackey MR, Perkins G, Ellisman MH, Latterich M, Schneiter R, Green DR and Newmeyer DD (2002) Bid, bax, and lipids cooperate to form supramolecular openings in the outer mitochondrial membrane. Cell 111: 331-342

14. Epand RF, Martinou J-C, Fornallaz-Mulhauser M, Hughes DW and Epand RM (2002) tBid promotes leakage by altering membrane curvature. J. Biol. Chem. 277: 32632-32639

15. Zha J, Weiler S, Oh K, Wei MC and Korsmeyer SJ (2000) Posttranslational Nmyristoylation of BID as a molecular switch for targeting to mitochondria and apoptosis. Science 290: 1761-1765

16. Degli Esposti M (2002) Lipids, cardiolipin and apoptosis, a greasy licence to kill. Cell Death Differ. 9: 234-236

17. Hardwick JM and Polster BM (2002) Bax, along with lipid conspirators, allows cytochrome $c$ to escape mitochondria. Mol. Cell 10: 963-965

18. Matsko CM, Hunter OC, Rabinowich H, Lotz, MT and Amoscato AA (2001) Mitochondrial lipids alterations during Fas- and radiation-induced apoptosis. Biochem. Biophys. Res. Commun. 287: 1112-1120

19. Ott M, Robertson JD, Gogvadze V, Zhivotosky B and Orrenius S (2002) Cytochrome $c$ release from mitochondria proceeds by a two-steps process. Proc. Natl. Acad. Sci. USA 99: 1259-1263

20. McMillin JB and Dowan W (2002) Cardiolipin and apoptosis. Biochim. Biophys. Acta 1585: $97-107$

21. Ma BJ, Taylor WA, Dlinsky VW and Hatch GM (1999) Acylation of monolysocardiolipin in rat heart. J. Lipid Res. 40: 1837-1845

22. Schlame M, Rua D and Greenberg ML (2000) The biosynthesis and functional role of cardiolipin. Prog. Lip Res. 39: 257-288

23. Tremblay JM, Helmkamp GM and Yarbrough LR (1996) Limited proteolysis of rat phosphatidylinositol transfer protein by trypsin cleaves the $C$ terminus, enhances binding to lipid vesicles, and reduces phospholipid transfer activity. J. Biol. Chem. 271: 21075-21080

24. Douliez JP, Jegou S, Pato C, Molle D, Tran V and Marion D (2001) Binding of two mono-acylated lipid monomers by the barley lipid transfer protein, LTP1, as viewed by fluorescence, isothermal titration calorimetry and molecular modelling. Eur. J. Biochem. 268: 384-388

25. Frolov A and Schroeder F (1998) Acyl coenzyme A binding protein. Conformational sensitivity to long chain fatty acyl-CoA. J. Biol. Chem. 273: 11049-11055

26. Richieri GV, Ogata RT and Kleinfeld AM (1992) A fluorescently labeled intestinal fatty acid protein. J. Biol. Chem. 267: 23495-23501

27. Thumser AEA, Voysey JE and Wilton DC (1994) The binding of lysophospholipids to rat liver fatty acid-binding protein. Biochem. J. 301: $801-806$

28. Arnold M, Ringler P and Brisson A (1995) A quantitative electrophoretic migration shift assay for analyzing specific binding of proteins to lipid ligands in vesicles and micelles. Biochim. Biophys. Acta 1233: 198-204

29. Hsu YT and Youle RJ (1997) Nonionic detergents induces dimerization among members of the Bcl-2 family. J. Biol. Chem. 272: 13829-13834

30. Schlame M and Rustow B (1990) Lysocardiolipin formation and reacylation in isolated rat liver mitochondria. Biochem. J. 272: 589-595

31. Yin XM, Wang K, Gross A, Zhao Y, Zinkel S, Klocke B, Roth KA and Korsmeyer SJ (1999) Bid-deficient mice are resistant to Fas-induced hepatocellular apoptosis. Nature 400: 886-891

32. Li S, Zhao Y, He X, Kim TH, Kuharsky DK, Rabinowich H, Chen J, Du C and Yin XM (2002) Relief of extrinsic pathway inhibition by the Bid-dependent mitochondrial release of Smac in Fas-mediated hepatocyte apoptosis. J. Biol. Chem. 277: 26912-26920

33. Tafani M, Karpinich NO, Hurster KA, Pastorino JG, Schneider T, Russo MA and Farber JL (2002) Cytochrome $c$ release upon Fas receptor activation depends on translocation of full-length bid and the induction of the mitochondrial permeability transition. J. Biol. Chem. 277: 10073-10082

34. Sarig R, Zaltsman Y, Marcellus RC, Flavell R, Mak TW and Gross A (2003) BID-D59A is a potent inducer of apoptosis in primary embryonic fibroblasts. J. Biol. Chem. 278: 10707-10715

35. George P, Ardail D, Rey C, Louisot P and Levrat C (2001) Phospholipids reacylation and palmitoylCoA control tumor necrosis factor- $\alpha$ sensitivity. Cytokine 13: 257-263

36. Cifone MG, Roncaioli P, De Maria R, Camarda G, Santoni A, Ruberti G and Testi R (1995) Multiple pathways originate at the Fas/APO-1 (CD95) receptor: sequential involvement of phosphatidylcholine-specific phospholipase $C$ and acidic sphingomyelinase in the proagation of the apoptotic signal. EMBO J. 14: 5859-5868

37. Reers M and Pfeiffer DR (1987) Inhibition of mitochondrial phospholipase $A 2$ by mono- and dilysocardiolipin. Biochemistry 26: 8038-8041

38. Atsumi G, Tajima M, Hadano A, Nakatani Y, Murakami M and Kudo I (1998) Fas-induced arachidonic acid release is mediated by Ca2+-independent phospholipase A2 but not cytosolic phospholipase A2, which undergoes proteolytic inactivation. J. Biol. Chem. 273: 13870-13877

39. Desagher S, Osen-Sand A, Montessuit S, Magnenat E, Vilbois F, Hochmann A, Journot L Antonsson B and Martinou J-C (2001) Phosphorylation of Bid by casein kinases I and II regulates its cleavage by caspase 8 . Mol. Cell 8 : 601-611

40. Petkovic M, Schiller J, Muller M, Bernard S, Reichl S, Arnold K and Arnhold J (2001) Detection of individual phospholipids in lipid mixtures by matrix-assisted laser desorption/ionization time-of-flight mass spectrometry. Anal. Biochem. 289: 202-216

41. Hendrickson HS, Hendrickson EK, Johnson ID and Farber SA (1999) Intramolecularly quenched BODIPY-labeled phospolipid analogs in phospholipase A2 and platelet-activating factor acetylhydrolase assays and in vivo fluorescence imaging. Anal. Biochem. 276: 27-35

42. Samworth CM, Degli Esposti M and Lenaz G (1988) Quenching of the intrinsic tryptophan fluorescence of the mitochondrial ubiquinol-cytochrome-c reductase by the binding of ubiquinone. Eur. J. Biochem. 171: 81-86 